\title{
Zuverlässigkeit von chromogenen Bestimmungen der Antithrombin III-Aktivität mit dem Behring Coagulation System
}

\author{
Reliability of Chromogenic Determinations of Antithrombin III-Activity with the Behring \\ Coagulation System
}

\author{
Ruth A. Fischer-Bieniek ${ }^{1,2}$, Th. Eller ${ }^{1}$, L. Volbracht ${ }^{1}$, D. Paar ${ }^{1}$
}

\begin{abstract}
Zusammenfassung: Die Zuverlässigkeit von chromogenen Bestimmungen der Antithrombin III-Aktivität mit dem vollmechanisierten Gerinnungsanalyzer Behring Coagulation System (BCS) wird im vorliegenden Beitrag beschrieben. Das von uns für das BCS adaptierte funktionelle Verfahren basiert auf der Reaktion von AT IIl mit dem Faktor Xa. Die ermittelte Impräzision mit Variationskoeffizienten von $2,2 \%$ und $3,7 \%$ in der Serie im normalen und klinisch bedeutsamen erniedrigten Bereich und von $4,3 \%$ und $5,2 \%$ von Tag zu Tag belegt die gute Präzision. Die ermittelten relativen Unrichtigkeiten betrugen bei Zielwerten von 95,5\% bzw. 56,8\% für die Antithrombin III-Aktivität $3 \%$ bzw. 4\%, was die gute Richtigkeit der Methode anzeigt. Im Vergleich zu zwei weiteren Analyzern, dem RA-XT ${ }^{\mathrm{TM}}$ und dem ACP 5040, wurde eine gute Übereinstimmung der Ergebnisse gefunden. Die Korrelationskoeffizienten lagen bei $0,96(n=56)$ und 0,98 $(n=49)$. Mit dem neuen vollmechanisierten BCS steht ein offenes System zur Verfügung, das die Integration von zuverlässigen chromogenen Antithrombin IIII-Bestimmungen in das Gerinnungslabor ermöglicht.
\end{abstract}

Schlüsselwörter: Antithrombin III; Blutgerinnungstests/Geräte; Chromogene Verbindungen; Reproduzierbarkeit der Ergebnisse.

Summary: The report describes the reliability of chromogenic determinations of antithrombin III (AT III) activity with the fully mechanized Behring Coagulation System (BCS). The procedure adapted on the $\mathrm{BCS}$ is based on the reaction of AT III with factor Xa. The good precision of the method is shown by an intra assay CV of $2.2 \%$ in the normal range and of $3.7 \%$ in the clinically important low range; interassay CVs were $4.3 \%$ and $5.2 \%$, respectively. The relative inaccuracy was $3 \%$ or $4 \%$ using control samples with assigned AT III activities of 95.5 and $56.8 \%$, showing

\footnotetext{
${ }^{1}$ Abt. für Klinische Chemie und Laboratoriumsdiagnostik, Zentrum für Innere Medizin, Universitätsklinikum Essen

${ }^{2}$ Korrespondenzadresse: Dr. Ruth A. Fischer-Bieniek, Abteilung für Klinische Chemie und Laboratoriumsdiagnostik, Zentrum für Innere Medizin, Universitätsklinikum Essen, Hufelandstraße 55, D-.45122 Essen. Fax: +49-201-723-5975

Eingegangen: 21. Oktober 1996 / Angenommen: 15. Januar 1997
}

the good accuracy of the method. In comparison studies with two other analyzers, the RA-XTTM and the ACP 5040, we found a good agreement of results; the coefficients of correlation were about $0.96(n=56)$ and $0,98(n=49)$. The Behring Coagulation System represents a new fully mechanized open system, which allows reliable chromogenic antithrombin III-determinations in the coagulation laboratory.

Keywords: Antithrombin III; Blood Coagulation Tests/instrumentation; Chromogenic Compounds; Reproducibility of Results.

$D^{2}$ a Antithrombin III (AT III) einer der wichtigsten plasmatischen Proteaseinhibitoren ist, hat die kontinuierliche Überwachung der AT III-Aktivitäten vor allem in der Intensivmedizin große Bedeutung gewonnen [1,2]. Die primär entwickelten Bestimmungsverfahren basieren auf der Anti-Faktor IIa-Aktivität des AT III. Da aber die gemessenen AT III-Aktivitäten auch von der Konzentration des Heparin-Cofaktors II abhängig sind, wurden in den letzten Jahren Testsysteme entwickelt, die die Anti-Faktor Xa-Aktivität des AT III nutzen $[3,4,5]$. Diese Untersuchungsmethoden sind unabhängig von der Heparin-Cofaktor II-Konzentration und spiegeln nur die AT III-Aktivität einer Plasmaprobe wider [6,7]. Wir berichten über Untersuchungen zur Zuverlässigkeit von chromogenen Bestimmungen [8] der AT III-Aktivität mit dem Behring Coagulation System (BCS, Behring Diagnostics GmbH, Marburg). Das von uns für das BCS adaptierte funktionelle Verfahren basiert auf der Reaktion von AT III mit dem Faktor Xa (FXa). Im einzelnen prüften wir die Impräzision, die Richtigkeit sowie die Vergleichbarkeit der erhaltenen Ergebnisse des BCS mit denen zweier anderer von uns routinemäßig eingesetzten mechanisierten Analysensystemen.

\section{Material und Methoden}

\section{Reagenzien}

Testkit: Coamatic $®$ AT 400 (Fa. Chromogenix AB, Mölndal, Schweden): Das Substrat S-2772 wurde in 
Tabelle 1 Pipettierschema für die Bestimmung der AT III-Aktivität mit dem Coamatic@ AT 400-Test auf dem BCS

1 Probentransferarm Dest. Wasser aufnehmen Probe aulnehmen Abgabe Küvette außen

2 Reagenztransferarm Luft autnehmen Reagenz aufnehmen Abgabe Küvette innen

3 Reagenztransferarm Luft aufnehmen Reagenz aufnehmen Abgabe Küvette Mitte

\section{Waschen Typ 4 $<$ kein Reagenz> <kein Reagenz> <kein Reagenz>}

Waschen Typ 2 $<$ kein Reagenz> F-Xa-Reagenz <kein Reagenz>

Waschen Typ 5 <kein Reagenz> AT-400 Substrat <kein Reagenz>

Mischaktion 2
Pipettierprofil 3
Pipettierprofil 3
Pipettierprofil 3
Mischen nach Inkubation
Pipettierprofil 3
Pipettierprofil 3
Pipettierprofil 3
keine Mischrotation
Pipettierprofil 3
Pipettierprofil 3
Pipettierprofil 3

0 Sek

o Sek.

$20 \mu l$

$5 \mu$

$25 \mu 1$

180 Sek. 240 Sek

$20 \mu \mathrm{l}$

$225 \mu l$

$225 \mu$

0 Sek. 0 Sek.

$20 \mu l$

$50 \mu l$.

$50 \mu$

\section{BCS \\ Name der Methode \\ Wellenlänge \\ Photometeraussteuerung \\ Enzymleerwert \\ 1-fach-Bestimmung bei Probenmessungen \\ 2-fach-Bestimmung für Kalibrationen \\ zulässige relative Abweichung bei Mehrfachbestimmung \\ Verdünnungsfaktor \\ Verdünnungsmedium \\ AT ill \\ $405 \mathrm{~nm}$ \\ $0^{\circ}-100 \mathrm{mE}$ \\ keiner \\ $10 \%$ \\ $1: 5$ \\ $\mathrm{NaCl}$}

Tabelle 2 Methoden-Parameter für die Bestimmung der AT Ill-Aktivität mit dem Coamatic® AT 400-Test auf dem

$17 \mathrm{ml}$ sterilem, destilliertem Wasser und der $\mathrm{F} \mathrm{Xa} \mathrm{in} 25$ ml Puffer, pH-Wert 8,2, gelöst.

Kalibrator: Standard Human Plasma (Behring Diagnostics $\mathrm{GmbH}$, Marburg)

Kontrollmaterial: PreciChrom ${ }^{\circledR}$ I/II (Boehringer Mannheim): Zielwerte 95,5\%/56,8\%, Bereiche 75,4$116 \% / 44,9-68,7 \%$

\section{Patientenproben}

Als Untersuchungsmaterial dienten Citratplasmaproben $(1$ Teil Trinatriumcitrat $0,106 \mathrm{~mol} / 1$ und 9 Teile Venenblut, S-Monovette ${ }^{\circledR}$ Sarstedt, Nümbrecht; Zentrifugation 10 Minuten bei $2000 \times \mathrm{g}$ ) von Patienten des Universitätsklinikum Essen, bei denen im Rahmen der routinemäßigen Gerinnungsanalytik die Bestimmung der AT III-Aktivität gewünscht wurde.

Zur Bestimmung der Impräzision in der Serie wurden 2 Plasmapools sowohl im hochnormalen (135\%), als auch im klinisch bedeutsamen pathologisch niedrigen $(58 \%)$ AT III-Aktivitätsbereich von jeweils 20 Plasmaproben verwendet. Für die Vergleichsmessungen wurden 105 Plasmaproben eingesetzt. Sämtliche Untersuchungen erfolgten als Einfachbestimmungen.

Nicht standardisierte Abkürzungen: AT III, Antithrombin III; FXa, aktivierter Gerinnungsfaktor X.

\section{Analysenprinzip}

Die Plasmaprobe wird mit einem Überschuß an FXa und in Gegenwart von Heparin inkubiert. AnschlieBend wird die verbleibende FXa-Aktivität über die Hydrolyserate des chromogenen Substrates S-2772 bestimmt. Die bei $405 \mathrm{~nm}$ gemessene Freisetzung von pNitroanilin ist umgekehrt proportional der AT III-Aktivität der Probe.

\section{Testdurchführung}

Für die Messungen wurden neben dem BCS das RA$\mathrm{XT}^{\mathrm{TM}}$-System (Bayer Diagnostics, München) und das ACP 5040-System (Eppendorf-Netheler-Hinz, Hamburg) eingesetzt. Bei den beiden letztgenannten Analysengeräten erfolgten die AT III-Bestimmungen ebenfalls mit dem Coamatio @ AT 400-Testkit.

Das BCS ist ein Zentrifugalanalyzer, der gleichzeitig chromogene, koagulometrische und immunologische Testverfahren mit positiver Probenidentifikation durchführen kann [9]. Die Küvettenrotoren des BCS werden mit Hilfe eines Rotorarms in die unterschiedlichen Positionen, wie Inkubations-, Meß-, Vorrats- und Abfallposition gebracht. Durch diese unterschiedlichen Positionen können gleichzeitig mehrere Arbeitsschritte (Bestückung und Entladung der Rotoren, Pipettierungen u.a.) durchgeführt werden. Während́d der Zentrifugation können bis zu 20 
Tabelle 3 Impräzision von Bestimmungen der AT III-Aktivität in der Serie mit dem BCS unter Verwendung von zwei Plasmapools (jeweils $n=10$ )

\begin{tabular}{llll}
\hline Mittelwert (Norm-\%) & Anzahl & Standardabweichung (Norm-\%) & Variationskoeffizient (\%) \\
135 & 10 & 2,99 & 2,2 \\
58 & 10 & 2,13 & 3,7
\end{tabular}

Tabelle 4 Impräzision von Bestimmungen der AT III-Aktivität von Tag zu Tag unter Verwendung der Kontrollplasmen PreciChrom® | und II

$\begin{array}{llll}\text { Mittelwert (Norm-\%) } & \text { Anzahl } & \text { Standardabweichung (Norm-\%) } & \text { Variationskoeffizient (\%)' } \\ 98 & 20 & 4,20 & 4,3 \% \\ 59 \% & 20 & 3,08 & 5,2 \% .\end{array}$

Tabelle 5 Daten der Regressionsanalyse [y=a+bx] nach Passing und Bablok [10] und Korrelationskoeffizienten für die mit den verschiedenen Analysensystemen erhaltenen AT III-Aktivitäten

\begin{tabular}{lllll}
\hline X/Y & Anzahl $n$ & Achsenabschnitt a (Norm-\%) & Steigung b & Korrelationskoeffizient $r$ \\
RAXT / BCS & 49 & 1,2 & 0,93 & 0,983 \\
ACP / BCS & 56. & 0,0 & 1,0 & 0,961
\end{tabular}

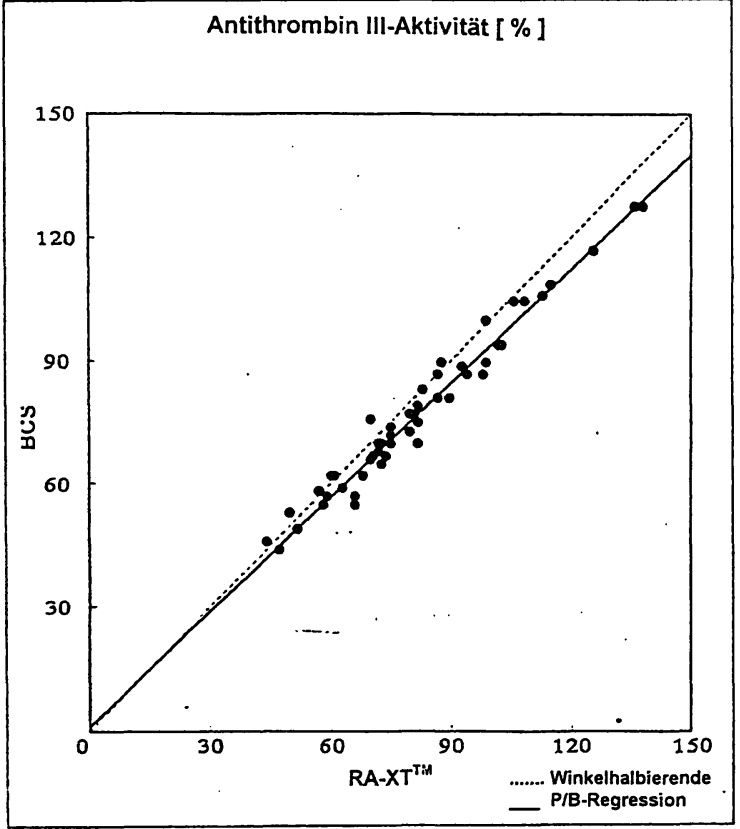

Abbildung 1 Vergleich von AT. !ll-Aktivitätsbestimmungen mit dem RA-XTTM und BCS

verschiedene Teste gleichzeitig verfolgt werden. Das System verfügt über 2 Pipettierarme für Proben und Reagenzien.

Bei der AT III-Aktivitätsbestimmung wird nach einer maximalen Zeit von 60 Sekunden bei einer Wellenlänge von $405 \mathrm{~nm}$ die Extinktion gemessen und aus dieser Kinetik die Steigung ermitteli (Delta E/min). Das Ergebnis wird anhand einer Eichkurve in \% der

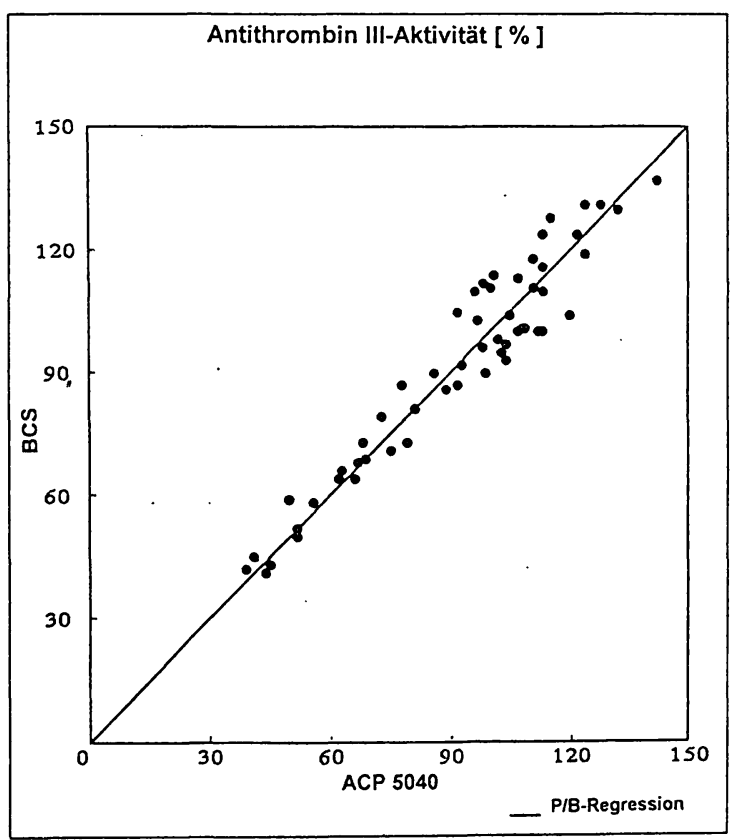

Abbildung 2 Vergleich von AT III-Aktivitätsbestimmungen mit dem ACP 5040 und BCS

Norm angegeben. Zur Erstellung der Eichkurve wird der Kalibrator Standard Human Plasma verwendet: unverdünnt $(100 \%)$, Verdünnungen mit $0,154 \mathrm{~mol} / \mathrm{l} \mathrm{Na}$ triumchloridlösung: $75 \%$, $50 \%$ und $25 \%$.

Die Ermittlung der AT III-Aktivitäl erfolgte am BCS nach dem Pipettierschema in Tab. 1. Weitere methodenspezifische Parameter der adaptierten AT IIIBestimmung sind in Tab. 2 aufgeführt. 


\section{Statistik}

Dic Regressionsanalyse der Daten der Methodenvergleiche wurde nach Pussing und Bablok vorgenommen โ10].

\section{Ergebnisse}

Es fanden sich bei der Messung in der Serie Impräzisionen zwischen 2,2 und 3,7\% (Tab. 3), bei der Messung von Tag zu Tag zwischen $4,3 \%$ und $5,2 \%$ (Tab. 4). Bei Zielwerten von $95,5 \%$ bzw. $56,8 \%$ für die AT Ill-Aktivität in den Kontrollproben PreciChrom® I bzw. II lagen die relativen Unrichtigkeiten im Mittel bei $3 \%$ bzw. $4 \%$.

Bei den Vergleichsuntersuchungen fand sich, wie aus den Abbildungen 1 und 2 und aus den Korrelationsdaten der Tabelle 5 hervorgeht, eine lineare Korrelation zwischen den Ergebnissen der AT III-Aktivitätsbestimmungen auf dem BCS, sowohl im Vergleich zum RA-XT'M als auch zum ACP 5040-System.

\section{Diskussion}

Für die hämostaseologische Notfalldiagnostik ist es von großem Vorteil, vollmechanisierte Meßgeräte zur Verfügung zu haben $[11,12,13]$. Deshalb wurde die auf der Anti-Faktor Xa-Aktivität beruhende, nicht durch Heparin beeinflußte [6] chromogene AT III-Aktivitätsmessung, die unabhängig von der Heparin-Cofaktor IIKonzentration ist, auf das BCS-System adaptiert. Bei den vorliegenden Untersuchungen wies diese Methode mit Variationskoeffizienten von 2,2 und $3,7 \%$ in der Serie bzw. 4,3 und 5,2\% von Tag zu Tag eine zufriedenstellende Präzision im hochnormalen, im normalen und im klinisch bedeutsamen niedrigen Bereich auf. Die geringen relativen Abweichungen vom Zielwert mit 3\% im normalen und $4 \%$ im Aktivitätsbereich von 55 bis $60 \%$, also in einem Bereich, der klinisch für den betroffenen Patienten ein potentielles Risiko zur Gerinnungsaktivierung und oder Thrombose bedeuten kann, belegen die gute Richtigkeit der Bestimmungen, soweit dies in der Hämostaseologie möglich ist. Die Korrelationskoeffizienten von 0,98 bzw. 0,96 bei den Vergleichsuntersuchungen von 105 Patientenproben zeigen die gute bis zufriedenstellende Vergleichbarkeit zwischen den mit dem BCS und dem RA-XT'M - bzw. ACP 5040-System bestimmten AT III-Aktivitäten.

Es steht somit mit dem neuen vollmechanisierten BCS ein offenes System zur Verfügung, das die Integration von zuverlässigen chromogenen AT IIIBestimmungen in das Gerinnungslabor ermöglicht.

\section{Literatur}

1. Schrader J, Züchner C, Köstering H, Schcler F. Chromogene Substratmethoden zur Antithrombin III-Bestimmung. Ärztl Lab 1986;32:111-4

2. Scherer R, Paar D, Stöcker L, Kox WJ. Diagnose und Therapie pathologischer Gerinnungsaktivierungen. Anästhesist 1994;43:347-54.

3. Tran TH. Duckert F. Influence of Heparin cofactor II (HCII) on the determination of Antithrombin III (AT).Thromb Res 1985;40:571-6.

4. Gram J, Jespersen J. Increased Concentrations of Heparin Cofactor II in Diabetic Patients, and Possible Effects on Thrombin Inhibition Assay of Antithrombin III. Clin Chem 1989;35:52-5.

5. Andersson NE, Menschik M, Van Voorthuizen H. New chromogenic AT III activity kit which is sensitive to heparin cofactor II and designed for use on automated instruments. Thromb Haemostas $1991 ; 65: 921$.

6. Bohner J, von Pape KW, Blaurock M.Thrombin-based Antithrombin Assays show overestimation of Antithrombin III Activity in Patients on Heparin Therapy due to Heparin Cofactor II Influence. Thromb and Haemostas 1994;71:280-3.

7. Bohner J, von Pape KW, Blaurock M. Chromogener Substrattest zur Bestimmung von Antithrombin III unter Verwendung des Faktors Xa. Lab med 1991;15:296-8.

8. Witt I. Test systems with Synthetic Peptide Substrates in Haemostaseology. Eur J Clin Chem Clin Biochem 1991;29: 355-74.

9. Stolz H, Keller F. Die Gerinnungssystem-Analyzer BehringCoagulation-Timer (BCT) und Behring-Coagulation-System (BCS). Neue Aspekte der Hämostaseologie. Diagnostik aktuell. Marburg: Med. Verlagsgesellschaft, 1996:14-23.

10. Passing $\mathrm{H}$, Bablok W. A new biometrical procedure for testing the equality of measurements from two different analytical methods. Application of linear regression procedures for method comparison studies in clinical chemistry. Part I. J Clin Chem Clin Biochem 1983;21:709-20.

11. Ziemer S, Hanke T, Teipel I, Engelhardt W, Fickenscher $K$. Evaluation of Fibrintimer A, a New, Fully Automated Coagulation Analyzer. Part II: Evaluation of Single Coagulation Factors and of Antithrombin III (AT III), Protein C and Protein S. Klin Lab 1995;41:21-29.

12. Paar D. Fully Mechanized Systems for Coagulation Testing. Eur J Clin Chem Clin Biochem 1995;33:A164.

13. Houbouyan L, Boutiere B, Contant G, Dautzenberg MD, Fievet P, Potron G, Vassault A, Gourmelin Y. Validation protocol of analytical hemostasis systems: measurement of anti-Xa activity of low-molecular-weight heparins. Clin Chem 1996;42: 1223-30. - 


\section{Neue Software zur Kostenkontrolle in medizinischen Laboratorien von Klinik und Praxis}

York Schmitt und Thorsten Schulz

D ie Notwendigkeit der Kostenkontrolle und nach Möglichkeit der Kostenreduktion ist vor allem in den medizinischen Laboratorien unbestritten. Der Kostendruck führt zu einem ökonomischen Handlungszwang mit eigenständiger Budgetverwaltung, für die verstärkt betriebswirtschaftliche Kenntnisse erforderlich sind. Dazu gehört auch die Einführung von EDVSystemen zum umfassenden Kosten-Controlling. Erstmalig zur Medica 1996 wurde eine neuartige Software präsentiert, die sämtliche medizinischen und betriebswirtschaftlichen Voraussetzungen und Bedürfnisse berücksichtigt. Das Programm wurde mit Unterstützung von Becton Dickinson in enger Zusammenarbeit zwischen einem Informatiker, einem Betriebswissenschaftler und einem Arzt für Laboratoriumsmedizin erarbeitet.

Der politische Wille zur Kostenreduktion im Gesundheitswesen kommt $u$. a. in den verschiedenen Gesundheitsstruktur-Reformgesetzen der Jahre 1993 bis 1996 und als Folge der anstehenden Ergänzungen der Gesetzgebung zum Ausdruck. Die Einführung neuer Gebührenordnungen für den kassen- sowie den privatärztlichen Bereich verstärkt diese Tendenz. Hinzu kommt die weitverbreitete Diskussion der Ausgliederung von Krankenhausinstituten oder der Fremdvergabe dieser Leistungen $\bar{a} \hat{n}^{-}$externe, niedergelassene Laboratorien

Die Programmziele der neuartigen Software zur Kostenkontrolle in medizinischen Laboratorien, die am Klinikum Darmstadt von den Autoren entwickelt und erstmalig auf der diesjährigen Medica vorgestellt wurde, sind die Kostenermittlung und Kostenbewertung.

Nach Erfassung aller direkten und indirekten Personalkosten einschließlich der gesamten Sachkosten liefert das Programm die detaillierten Kosten pro Ko-

Institut für Labormedizin am Klinikum Darmstadt Grafenstraße 9, D-64283 Darmstadt

Tel.: $06151 / 107-6300$ * Fax: $06151 / 107-6397$ stenträger, d. h., es werden für jeden erstellten Parameter die Kosten pro befundetem Parameter ermittelt. Auch eine Unterteilung nach Erbringung der Leistungen innerhalb bzw. außerhalb des Routinebetriebes ist möglich. Somit kann auch eine Deckungsbeitragsrechnung unter Berücksichtigung der möglichen Einnahmen aus den stationären und ambulanten Bereichen mit der Möglichkeit der Erstellung einer klaren Kostenstruktur durchgeführt werden. Dadurch wird z. B. die Erstellung von Angeboten für externe Auftraggeber erleichtert.

Ein besonderer Vorteil des Programms ist es, da $\beta$ die direkten Personalkosten innerhalb und außerhalb des Routinebetriebes nicht nur durch die sogenannte Arbeitsplatzmethode ermittelt werden ${ }^{*}$ können, die immer dann ungenau ist, wenn Geräte von verschiedenen Arbeitsplätzen gemeinsam genutzt werden. Die neue' Software bietet zusätzlich die Möglichkeit, auf ein neuartiges Konzept der Arbeitsgruppe „Analysenzeitermittlung" der Deutschen und Osterreichischen Gesellschaften für Klinische Chemie zurückzugreifen („Ermittlung des Personalbedarfs“, Instant-Schriftenreihe Band 8, Hg.: R. Haeckel, Springer-Verlag, Berlin 1992).

Weitere Anwendungsmöglichkeiten sind z. B. der Vergleich unterschiedlicher Rechnungsperioden sowie verschiedene statistische und grafische Darstellungen. Das Programm ermöglicht damit sowohl niedergelassenen als auch klinischen Laboratorien ein transparentes, auf einen Blick verständliches Kosten-Controlling. Dank des modularen Aufbaus und der Ausgabe der Ergebnisse in Listen ist es möglich, die erhaltenen Daten an die spezifischen Bedürfnisse der Anwender anzupassen und weiterzuverarbeiten.

Die Software läuft unter dem Programm Excel 5.0 auf jedem modernen PC. Sämtliche Programmabläufe wurden in der besonders anwenderfreundlichen Programmiersprache 'Visual Basis' erstellt. Neben der manuellen Eingabe aller notwendigen Daten ist auch die automatische Übernahme der notwendigen Datenlisten möglich, z. B. über eine Schnittstelle wahlweise mit Online-Datenübertragung oder über Diskette.

J Lab Med 1997; 21 (3): 173 


\section{Peter Krietsch/Manfred Dietel}

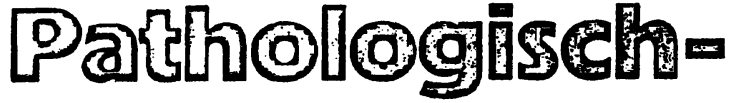

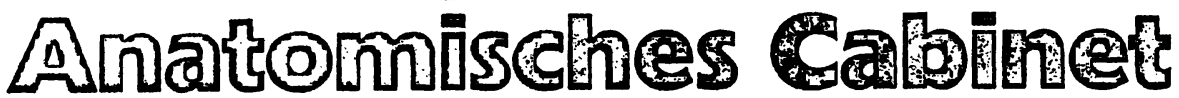

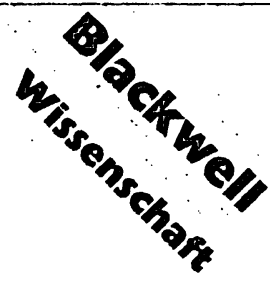

\section{Vom Virchow-Museum zum Berliner Medizinhistorischen Museum in der Charité}

1996. 192 Sciten mit 124 Farbabbildungen. $17 \times 24 \mathrm{~cm}$.

Broschiert. DM 48,-/öS 350,-/sFr 44,50 ISBN 3-89412-254-4

Medizinhistorisch hat Berlin Unvcrglcichliches zu bieten, brennpunktartig versammelt in der Charité: Hier arbeiteten die bedcutendsten Ärzte Deutschlands, hier war das Zentrum der Forschung, hier entstanden schon im 18. Jahrhundert anatomische Sammlungen, und hier eröffnete Rudolf Virchow 1899 sein Pathologisches Museum.

Dieses Buch präscnticrt das "Pathologisch-Anatomische Cabinet“ in dreierlei Sinn: Es folgt dem wechselvollen Schicksal der früher "Cabinet " genannten Sammlungen, erzählt die dramatischen Geschichten einiger erhalten gebliebener Exponatc und plädiert engagiert dafür, daß Berlin auch medizingeschichtlich wieder Hauptstadt wird durch ein Berliner Medizinhistorisches Museum.

"Die Geschichte dieser größten und wertvollsten Präparatesammlung, die jemals von einem Menschen zusammengetragen wurde, ist im vorliegenden, reich illustrierten Buch aufs lebendigste und mit vielen Quellenzitaten nachgezeichnet".

Frankfurter Allgemeine Zeitung

\section{Manfred Dietel/Peter Krietsch}

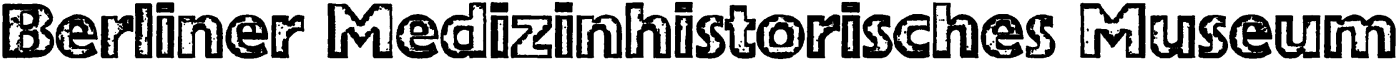

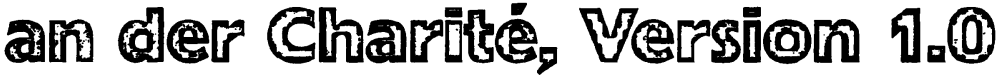

Systemmindestvoraussetzungen: 486 DX2/66, 4 MB Hauptspeicher, SVGA-Grafikkarte, 16-Bit-Soundkarte, Doublespeed CD-ROM-Laufwerk, DOS ab 5.0, Windows 3.X oder 95, Video für Windows, ca. 2 MB freien Festplattenspeicher. DM 98,-/öS 774,-/sFr 90,50 ISBN 3-89412-253-6

Sie finden auf dieser CD-ROM Exponate aus 300 Jahren Medizingeschichte. Sie sind ein Spiegelbild der Irrungen und Wirrungen der Natur mit Darstellungen von Fehlbildungen, Infektionskrankbeiten und Tumorleiden. Kurzbiographien bedeutender Wissenschaftler der Charité beschreiben deren Anstrengungen zur Erforschung der Krankheiten und zur Entwicklung angemessener Behandlungen. Ergänzend finden Sie eine Ausstellung des dafür notwendigen technischen Handwerkszeugs - von der ersten chirurgischen Säge über verschiedene Mikroskopgenerationen bis zu modernen Herzprothesen.

Die Museumsimagination, die Sie hier „betreten“ können, wurde in realitas 1899 von Rudolf Virchow eingeweiht und seiner Bestimmung als Heimstätte der weltweit einmaligen pathologisch-anatomischen Sammlung übergeben. Nach Beschädigung im Zweiten Weltkrieg und Fremdnutzung begann nach der Wiedervereinigung der steinige Weg der Rekonstruktion. Um auf diesem Weg vorangehen zu können, benötigt das Museum IHRE ideelle und materielle Hilfe. Diese CD-ROM soll dazu beitragen.

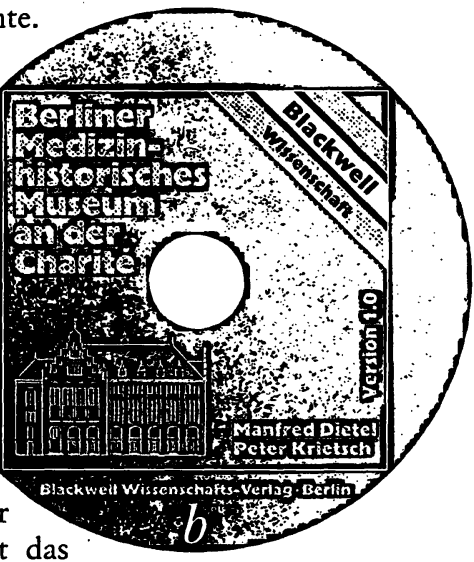

l'reisstand: 1. November 1996

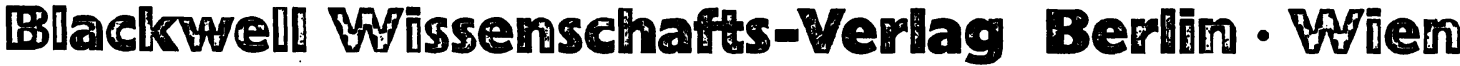

